\title{
Patientenprozesse als Leitlinie für die Teilzeitarbeit*
}

\author{
Jürg Unger-Köppel \\ Dr. med., Mitglied des FMH-Zentralvorstandes, Departementsverantwortlicher Stationäre Versorgung und Tarife
}

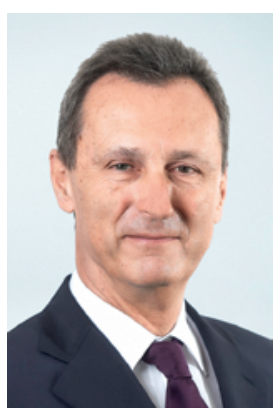

Wenige Chefärztinnen und Chefärzte können heute noch mit gutem Gewissen sagen, dass sie unter zahlreichen Bewerbenden die besten auswählen können. Das System ist vielerorts gekippt und der Stellenmarkt hat sich grundsätzlich verändert: Heute bewirbt sich die Institution beim Kandidaten. Wer in einem Markt ein Alleinstellungsmerkmal besitzt, hat grosse Vorteile, weil er Alleinanbieter ist. Dies zieht die Leute an und bindet sie. Attraktive Teilzeitarbeitsmöglichkeiten in einem guten Team können zu einem wesentlichen Anziehungspunkt auf dem Stellenmarkt werden. Vergesellschaftet mit einem guten Mentoring und einem tollen Weiterbildungsprogramm steigen die Chancen der Institution am Stellenmarkt deutlich.

Die Teilzeitarbeit ist in der Medizin längst angekommen. Wir müssen nicht mehr diskutieren, ob wir sie wollen oder nicht, sondern wir sollten gemeinsam die passenden Formen für die Patientenprozesse und die Mitarbeitenden finden. Teilzeitarbeit ist auch keine Geschlechterfrage, sondern eine Generationenfrage: Die Generation, welche die Zukunft in den Spitälern bestimmen wird, will oft Teilzeit arbeiten, und zwar Frauen wie Männer.

\section{Heute bewirbt sich die Institution beim Kandi- daten. Teilzeitarbeitsmöglichkeiten steigern die Chancen der Institution deutlich.}

Seit je ist bekannt, dass es Fächer gibt, in welchen die Teilzeitarbeit einfacher zu realisieren ist: Anästhesie und Kinder- und Jugendpsychiatrie werden dann meist aufgezählt. Neonatologie, Innere Medizin oder Chirurgie erscheinen am anderen Ende des Spektrums. Bereits bei der Wahl der Facharztausbildung kann man den Aspekt der Teilzeitarbeit miteinbeziehen. Der Weitblick sollte dabei über die Weiterbildungszeit hinausreichen und auch die Tätigkeit als Fachärztin oder Facharzt in der Institution oder der eigenen Praxis miteinbeziehen. Oft hört man, Teilzeitarbeit sei nur in den «kleinen Fächern» möglich, ohne dass man sich Rechenschaft gibt, dass die fortschreitende Spezialisierung auch in den "grossen Fächern» den Tätigkeitshorizont durch die Fokussierung einengt. Teilzeitarbeit ist sehr wohl möglich in den "grossen Fächern" oder in solchen, wo man dies bis vor kurzem abgelehnt hatte. Dazu zwei Beispiele: Ein Kaderärztinnenteam der Neonatologie will die Betreuungs- und Behandlungskonstanz mit Teilzeitarbeit kombinieren. Sie finden die Lösung in einem diachronen Teilzeitarbeitsmodell mit zwei 50\%-Pensen: Man arbeitet zwei Wochen 100\% und hat anschliessend zwei Wochen frei. So können sie dem Patientenprozess am besten gerecht werden. Anders ein nephrologisches Team, das für eine Dialysestation verantwortlich ist: Mit einem synchronen Teilzeitmodell, das sich am Rhythmus der Dialysen orientiert, arbeiten sie klassisch mit einem Wochentagsmodell, bei welchem man zum Beispiel am Montag, Mittwoch und Freitagmorgen tätig ist bei einem 50\%-Pensum.

Teilzeitarbeit ist längst in der Medizin angekommen. Wir sollten die passenden Formen für Patientenprozesse und Mitarbeitende finden.

Das zeigt, wie sich erfolgreiche Teilzeitarbeitsmodelle am Patientenprozess orientieren. In einem Universitätsbetrieb, wo Kongress- oder Publikationsfristen einzuhalten sind, gibt es weitere Optionen: Mit Jahresarbeitszeitmodellen kann vorübergehend mit einem höheren Pensum gearbeitet werden, um in der folgenden Zeit auch mehr Freizeit zu geniessen.

Und wie organisiert der Chef seine Klinik mit Teilzeitarbeitenden? Top down wird es kaum funktionieren. Zielführender ist es, jenen, die Teilzeitarbeit einführen wollen, die minimal notwendigen Rahmenbedingungen zu geben, so dass die Qualität der Patientenversorgung erhalten bleibt und die Rapporte nicht zunehmen. Dann ist die Kreativität der Beteiligten gefordert, die ja etwas für sich erreichen wollen und deshalb gerne Verantwortung übernehmen. So gelingen Prozesse viel leichter. Als Resultat sind die Mitarbeitenden zufriedener, was einen sehr hohen Wert darstellt, der letztlich auch wieder der Patientenversorgung zugute kommt. Und Teilzeitarbeitsmöglichkeiten in einem zufriedenen Team sind sehr wertvoll auf dem Arbeitsmarkt. 